\title{
Study of free-space coupling into mm-wave whispering-gallery mode resonators for a radioastronomy receiver
}

\author{
Gabriel Santamaría-Botello*, Kerlos Atia Abdalmalak*, Maria-Theresa Schlecht ${ }^{\dagger}$, David González-Ovejero ${ }^{\ddagger}$, \\ Florian Sedlmeir ${ }^{\S}$, Harald G. L. Schwefel ${ }^{\S}$, Stefan Malzer ${ }^{\dagger}$, Heiko Weber ${ }^{\dagger}$, Daniel Segovia-Vargas*, \\ Darragh McCarthy ", John Anthony Murphy ", Gottfried H. Döhler and Luis Enrique García Muñoz* \\ ${ }^{*}$ Universidad Carlos III de Madrid, Spain \\ legarcia@ing.uc3m.es \\ ${ }^{\dagger}$ Friedrich-Alexander Erlangen-Nürnberg University, Germany

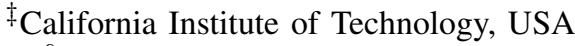 \\ $\S$ University of Otago, New Zealand \\ ฯ Maynooth University, Ireland
}

\begin{abstract}
In this paper, the coupling mechanism of a freespace Gaussian beam into a whispering-gallery mode resonator through a dielectric lens is mathematically modeled and numerically solved by means of the Schelkunoff-Waterman method (the so called T-matrix method). This approach allows in principle, to quickly analyze the performance of different near-field coupling mechanisms with arbitrary excitations. The aim is to efficiently excite a WGM into a nonlinear dielectric resonator in order to detect the weak mm-wave radiation from the cosmic microwave background (CMB) by up-converting the signal into the optical domain via the nonlinearity of the medium.
\end{abstract}

\section{INTRODUCTION}

The nonlinear up-conversion of mm-wave and sub-mmwave signals into the optical domain by means of the nonlinearity of high-quality WGM resonators is a promising technique for designing high-sensitivity receivers [1]. The intrinsically noiseless mechanism of the parametric up-conversion would allow these receivers to operate in a photon-counting regime at room temperature [2], which makes them attractive for radio-astronomy applications, as for example the accurate measurement of the CMB B-mode polarization for indirectly detecting primordial gravitational waves. This is a topic of high interest since it would test the validity of the inflationary paradigm. The general scheme of these receivers involves a WGM resonator in which an optical and a mm-wave WGMs are excited by near-field couplers. The optical WGM is typically excited by means of a coupling prism, whereas the $\mathrm{mm}$-wave signal is typically coupled through a dielectric rod waveguide which requires transitions and a horn antenna to be fed by a free-space Gaussian beam.

A free-space Gaussian beam can be coupled to spherical resonators if the beam is directed tangentially to the resonator's surface at an optimal distance, however the coupling efficiency is low [3]. An alternative is to couple the Gaussian beam to the resonator by the evanescent near-field arising from the planar face of a semi-spherical dielectric lens, when the beam is incident beyond the critical angle. This is the same principle used in the optical domain to couple the laser signal through a prism. Fig. 1 shows the general scheme of the receiver, depicting the coupling lens mechanism. In this case, an exhaustive analysis must be done in order to find the optimal parameters of the coupler which maximize the intracavity power.

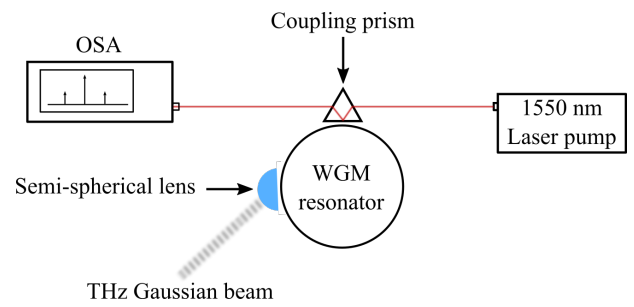

Fig. 1. General scheme for the receiver based on nonlinear up-conversion in a WGM resonator.

For quickly analyzing the system, we study the coupling mechanism by computing the evanescent field arising from the Gaussian beam under total internal reflection (TIR), and consider this as an incident primary field. Then, the coupled field inside the resonator is computed by means of the T-matrix method. This is a first approximation since the presence of the lens in the vicinity of the resonator is neglected from the formulation. As a second approximation we propose to compute the plane-wave spectrum of the fields, and consider the multiple reflections occurring between the resonator and the lens' face.

\section{Mathematical Framework}

The system is modeled as a planar interface between the lens medium with refractive index $n_{a}$ and the air $\left(n_{b}\right)$, which is at a distance $d$ from the resonator's center as shown in Fig. 2. Consider the incident field $\boldsymbol{E}_{a}^{i}\left(\boldsymbol{r}^{\prime}\right)$ in medium $n_{a}$ expanded 


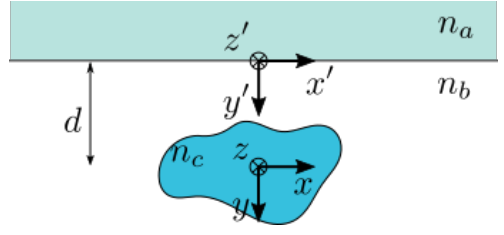

Fig. 2. Model of the system.

in a continuum of plane waves:

$$
\begin{array}{r}
\boldsymbol{E}_{a}^{i}\left(\boldsymbol{r}^{\prime}\right)=\iint\left[\Psi_{\perp}^{ \pm}\left(\kappa_{x}, \kappa_{z}\right)+\Psi_{\|}^{ \pm}\left(\kappa_{x}, \kappa_{z}\right) \hat{\boldsymbol{\kappa}}^{ \pm} \times\right] \hat{\boldsymbol{\kappa}}^{ \pm} \times \hat{\boldsymbol{a}}_{y} \\
\cdot \exp \left(i \kappa_{a} \hat{\boldsymbol{\kappa}}^{ \pm} \cdot \boldsymbol{r}^{\prime}\right) \mathrm{d} \kappa_{x} \mathrm{~d} \kappa_{z} \quad(1)
\end{array}
$$

where $\kappa_{a} \hat{\boldsymbol{\kappa}}^{ \pm}=\kappa_{x} \hat{\boldsymbol{a}}_{x} \pm \sqrt{\kappa_{a}^{2}-\kappa_{x}^{2}+\kappa_{z}^{2}} \hat{\boldsymbol{a}}_{y}+\kappa_{z} \hat{\boldsymbol{a}}_{z}$, and the functions $\Psi_{\perp}^{ \pm}$and $\Psi_{\|}^{ \pm}$are the plane-wave spectrum of the field for perpendicular and parallel polarization respectively, given by the two-dimensional Fourier transform of the field. To each plane wave of the integral, we can apply Snell's laws to obtain the spectrum of the reflected and refracted fields so we can also express them as a continuum of plane waves. Computationally we can project the resulting incident, reflected and refracted fields in a fixed set of plane wave directions forming an incomplete approximate basis of the whole vector space of field solutions. Then, each plane-wave component of the refracted field (for each polarization) can be arranged in a column vector $E_{\perp, \|}^{t}$ and obtained from a matrix multiplication with the column vector associated with the incident field expansion as $E_{\perp, \|}^{t}=T_{\perp, \|}^{a b} E_{\perp, \|}^{i}$, where the square matrix $T_{\perp, \|}^{a b}$ is obtained from Snell's laws. From now on, we will get rid of the polarization sub-index and recall that every calculation must be done for each polarization.

Once the refracted field is computed, it can be considered as a primary field incident into the scatterer. Since Maxwell's equations are linear, the refracted and scattered fields inside and outside the resonator, $E^{o}$ and $E^{s}$ respectively, can be thought as determined by some linear transformation applied to the incident field. Hence, these fields can be computed from the matrices associated with the transformations as $E^{o}=Q E^{r}$ and $E^{s}=S E^{r}$. Matrices $Q$ and $S$ are uniquely determined by the resonator's material and geometry, and may be obtained from the T-matrix method [4], recalling that incident plane waves must be expanded in vector spherical harmonics (VSH), and scattered VSH must be converted back into a plane-wave expansion [5], [6]. The refracted field inside the resonator $E^{o}$ can be considered as a first approximation of the excited field. The accuracy of the solution can be improved by taking into account the contribution of the multiple reflections between planar interface and resonator:

$$
\begin{aligned}
E^{o} & =E_{1}^{o}+E_{2}^{o}+E_{3}^{o}+\cdots \\
& =Q\left[I+R^{b a} S+\left(R^{b a} S\right)^{2}+\cdots\right] E_{1}^{t} \\
& =Q\left(I-R^{b a} S\right)^{-1} T^{a b} E^{i}
\end{aligned}
$$

where $I$ is the identity matrix, and $R^{b a}$ is a square matrix determined from Snell's laws that computes the reflection of plane waves coming from $n_{b}$ to $n_{a}$.

\section{RESULTS}

Preliminary results of the first approximation field excited in a sapphire sphere of radius $R=2.5 \mathrm{~mm}$ at $73.28 \mathrm{GHz}$ when a Gaussian beam is incident in a silicon lens under TIR have been obtained. The distance between the resonator's surface and the lens is $0.25 \lambda_{0}$, being $\lambda_{0}$ the free-space wavelength. An iterative study showed that it is optimal to have the beam focused in the point on the lens' flat face which is closest to the resonator. It was also shown that the optimal angle depends slightly on the beam waist. The maximum field intensity of a resonant TE mode inside the resonator was found for a beam waist $w_{0}=1.35 \lambda_{0} / n_{a}$ and an incidence angle $\gamma=29^{\circ}$. The excited field is plotted in Fig. 3, being the intensity about 6 times larger than in a free-space beam coupling scheme without lens, in the optimal configuration and for the same beam power.

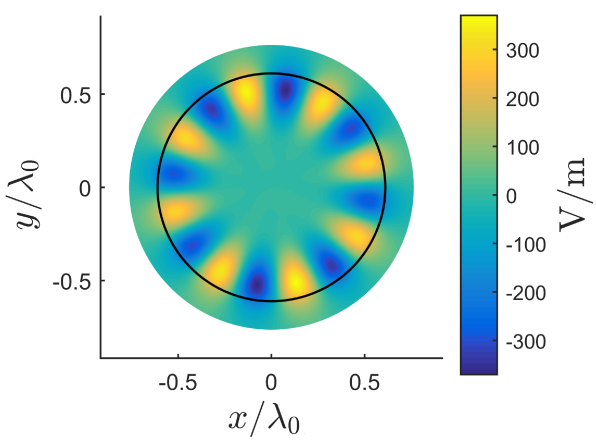

Fig. 3. Real part of the electric field evaluated on an azimuthal plane inside the sphere for the optimal configuration. The Gaussian beam's electric field magnitude inside the lens is $7.33 \mathrm{~V} / \mathrm{m}$.

\section{ACKNOWLEDGEMENT}

This work has been financially supported by "DiDaCTIC: Desarrollo de un sistema de comunicaciones inalambrico en rango THz integrado de alta tasa de datos", TEC201347753-C3, CAM S2013/ICE-3004 “DIFRAGEOS" projects, "Proyecto realizado con la Ayuda Fundación BBVA a Investigadores y Creadores Culturales 2016." and "Estancias de movilidad de profesores PRX16/00021".

\section{REFERENCES}

[1] R. W. Boyd, Nonlinear Optics. 2nd ed. 2003

[2] A.B Matsko, D. V. Strekalov and N. Yu, Sensitivity of THz photonic receivers, Physical Review A 77, 043812, 2008.

[3] James A. Lock, Excitation Efficiency of a Morphology-Dependent Resonance by a Focused Gaussian Beam, J. Opt. Soc. Am. Vol. 15, No. 12 , 1998.

[4] P. Barber, C. Yeh, Scattering of electromagnetic waves by arbitrarily shaped dielectric bodies, Applied Optics. 14, 12, 1975

[5] Stratton J. A., Electromagnetic Theory, McGraw-Hill, New York, 1941.

[6] J. E. Hansen, Spherical near-field antenna measurements, IEE Electromagnetic Waves Series, 26. 